\title{
Plasmonic Oligomers With Tunable Conductive Nanojunctions
}

\author{
Xiaoyan Li, ${ }^{1}$ Jieli Lyu, ${ }^{1}$ Claire Goldmann, ${ }^{1}$ Mathieu Kociak, ${ }^{1}$ Doru Constantin, ${ }^{1 *}$ Cyrille \\ Hamon $^{1 *}$ \\ ${ }^{1}$ Laboratoire de Physique des Solides, CNRS, Univ. Paris-Sud, Université Paris-Saclay, 91405 \\ Orsay Cedex, France.
}

\begin{abstract}
Plasmonic particles can be welded together, but controlling the metallurgy of the hotspots is a challenge in colloidal chemistry. In this paper, we demonstrate an original method that connects gold particles to their neighbors by another metal of choice. To achieve this goal, we first assemble gold bipyramids in a tip-to-tip configuration, yielding short chains of variable length. The good colloidal stability and surface accessibility make the nanochains suitable seeds to grow metallic junctions in a second step. We follow the oligomer formation and the deposition of the second metal (i.e. silver or palladium) via UV/Vis spectroscopy and we map the plasmonic properties of the nanostructures at nanometer scale using electron energy loss spectroscopy. The formation of silver bridges leads to a huge redshift of the longitudinal plasmon modes into the mid-infrared region, while the addition of palladium results in a redshift accompanied by significant plasmon damping.
\end{abstract}

\section{Table of content}

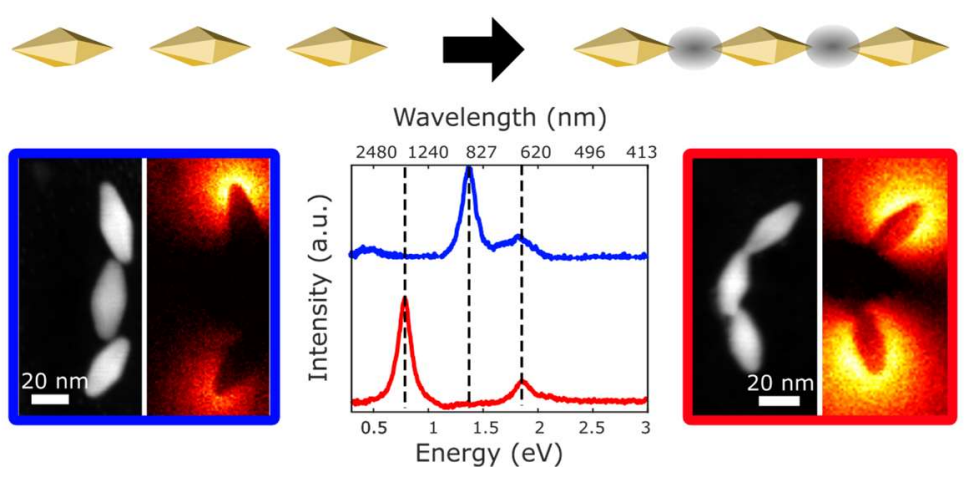


Noble metal nanoparticles (NPs) can support light-driven collective electron oscillations, known as localized surface plasmon resonances (LSPR), which can be tuned by the composition, shape and environment of the NPs. Plasmon hybridization can take place when NPs which sustain modes with close eigenergies enter into proximity so that the electromagnetic field of these modes overlap spatially, resulting in amplified electromagnetic fields at the junction between nanoparticles, the enhancement being more intense as the distance decreases. ${ }^{1-5}$ These regions of intense optical activity have led to applications (ranging from biosensing to metamaterial design) that strongly depend on the detailed geometry of the metal surfaces. $^{6-7}$

Controlling the shape and chemical nature of the optically actives areas (or hotspots) in colloidal plasmonic nanosystems has a very wide range of applications. For instance, one can tailor plasmon coupling by bridging nanoparticles together with molecular junctions whose conductivity can be switched at will. ${ }^{8-9}$ Whether nanoparticles are immobilized on a surface or in colloidal suspension, other strategies focus on controlling the metallurgy of the junctions. For instance, the conductivity of the junctions of gold nanoparticle dimers adsorbed on an electrode has been tuned by electrodeposition. ${ }^{10-11}$ Controlled melting of adjacent nanoparticles has been performed at the single particle level by an electron beam in order to connect gold nanospheres into solid networks featuring low-energy plasmon modes. ${ }^{12}$ Colloidal approaches include welding nanoparticle assemblies using ultrafast laser pulses. ${ }^{13-14}$ In another example, conductive connections were engineered by controlling the sintering of gold nanoparticle dimers within a silica shell. ${ }^{15}$ Another group has controlled the silver thickness at the junction of asymmetric dimers linked with DNA. ${ }^{16}$ Although some colloidal-based strategies have been demonstrated, engineering plasmonic assemblies with tunable conductive junctions remains challenging. Current methods rely on the controlled fusing or melting of nanoparticle 
assemblies but do not allow control over the metallic nature of the nanojunction. Moreover, most studies have focused on dimers rather than on larger objects, which are however more interesting for the directional control of light.

Scanning Transmission Electron Microscopy - Electron Energy Loss Spectroscopy (STEM-EELS) is an effective technique for interrogating the plasmonic response of individual and assembled nanoparticles, including one-dimensional chains. ${ }^{12,}$ 17-21 Hyperspectral measurements with a spatial resolution of a few nanometers enable the direct observation of plasmon modes. The nature of each plasmon mode can be determined by its spatial distribution and low loss energies: transverse $(\mathrm{T})$ and longitudinal (L) plasmon modes can thus be characterized. The notation $\mathrm{L}_{\mathrm{N}}$, where $\mathrm{N}-1$ stands for the number of charge nodes along the assembly direction is convenient to describe modes from the lowest to highest energy. One can distinguish the fundamental dipolar mode (L1), its harmonics $(\mathrm{N} \geq 2)$ as well as bright (odd $\mathrm{N}$ ) and dark plasmon modes (even N). ${ }^{19}{ }^{22-23}$ Moreover, plasmon mapping reveals the high symmetry of the modes across the nanostructures and the spatial distribution of the modes as well as the number of nodes further confirms the identification of $\mathrm{L}_{\mathrm{N} \geq 2}$ modes. This technique complements bulk UV/Vis spectroscopy measurements by studying in more detail the collective plasmonic properties of assembled species at the single particle level.

In this work, we developed a seed mediated growth procedure to deposit a metal of choice at the hotspots location in nanoparticles assemblies. We produced colloidally stable assemblies of gold bipyramids and then grew metallic patches at the nanoparticle junctions. We focused on silver and palladium due to their notable plasmonic and photocatalytic properties, respectively. UV/Vis spectroscopy and Electron Energy Loss Spectroscopy (EELS) were used to describe the evolution of the collective plasmonic response of the nanostructures. Upon bridging the nanoparticles we observed a strong variation of the optical properties, which can be tuned by the nature of the metallic junction. 
Elongated chain-like structures comprising separated nanoparticles are often referred to as plasmonic "polymers", in direct analogy with their molecular counterparts. ${ }^{5,24-25}$ In waterbased synthetic procedures, such objects are generally impractical for further use, due to the fast self-assembly kinetics (which leads to precipitation) and/or to surface passivation by thiolated ligands. A recent study provided an alternative to dithiolated linkers by showing that sulfate ions can mediate end-to-end attachment of gold nanorods. ${ }^{26}$ We built upon this study to assemble gold bipyramids (GBPs) oligomers and refined the process to better control the chain formation kinetics (Figure 1).

A Self assembly

Freeze

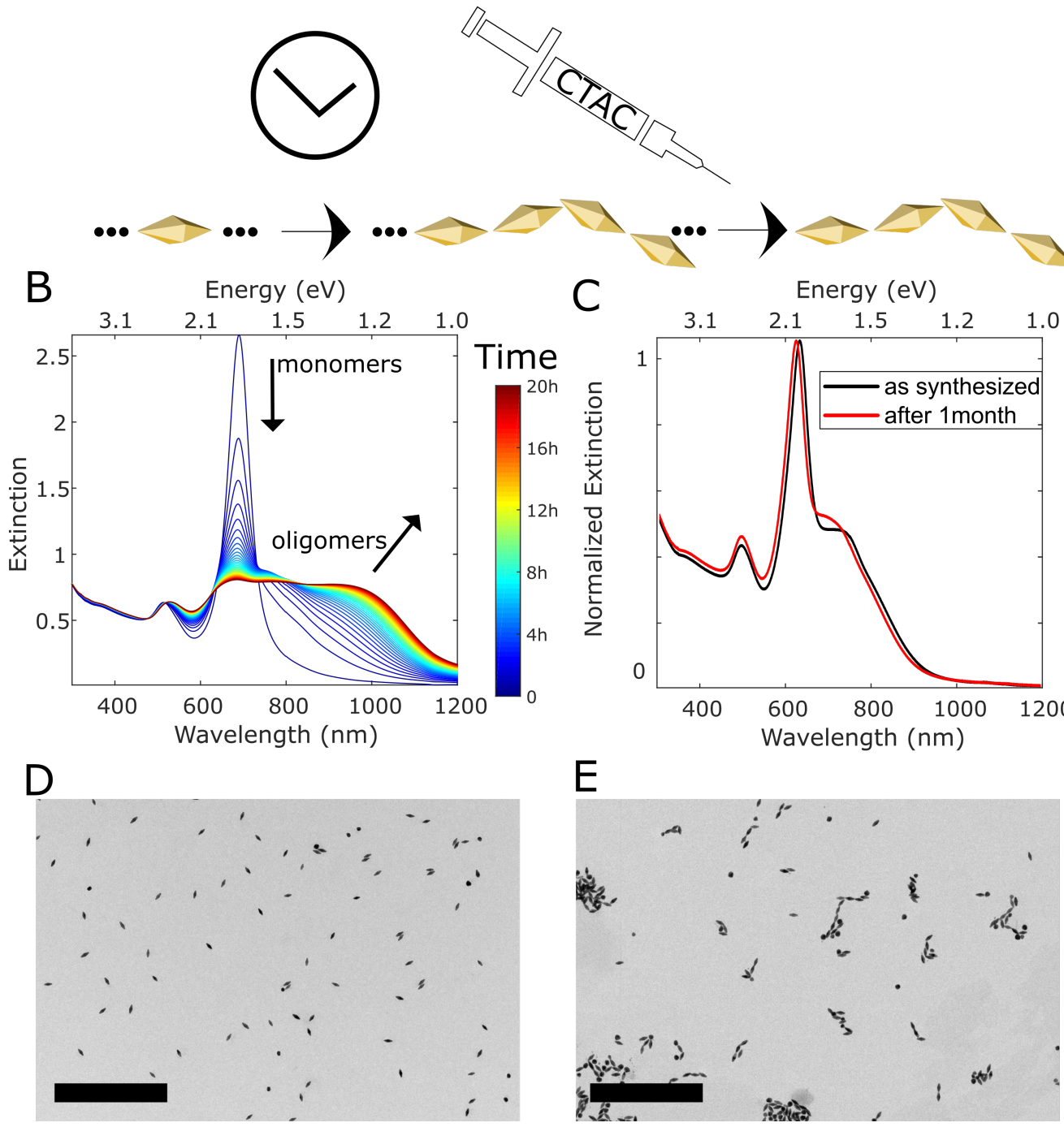


Figure 1: Chain formation and colloidal stabilization. A) Scheme illustrating the tip-to-tip assembly of GBPs mediated by sulfate ions and the process used to quench the system by the addition of CTAC. B) Series of extinction spectra during the assembly of GBPs $(29 \mu \mathrm{M}$ CTAB, $\left.0.25 \mathrm{mM} \mathrm{Au}^{0}\right)$ mediated by sulfate ions $(1 \mathrm{mM})$, acquired at time intervals of $30 \mathrm{~min} . \mathbf{C}$ ) Normalized UV/Vis spectra of the oligomers (incubated for 6h) immediately after supplementing the solution with $10 \mathrm{mM} \mathrm{CTAC} \mathrm{(black} \mathrm{line)} \mathrm{and} \mathrm{the} \mathrm{same} \mathrm{dispersions} \mathrm{after} 1$ month (red line). D-E) Representative low-magnification TEM images of GBPs (d) before and (e) after assembly (20h of reaction). Scale bar is $500 \mathrm{~nm}$.

The preparation and characterization of GBPs assemblies are described in the Supporting Information. In short, oligomers were obtained by introducing $1 \mathrm{mM} \mathrm{MgSO}$ into the GBPs suspension (Figure 1A). We monitored the evolution of the optical properties by means of time-resolved UV/Vis spectroscopy (Figure 1B). The intensity of the longitudinal surface plasmon resonance (LSPR) band at $696 \mathrm{~nm}$ intensity drops over $20 \mathrm{~h}$ while the transversal surface plasmon resonance (TSPR) band at $510 \mathrm{~nm}$ did not change noticeably. Simultaneously, a new plasmon band appears which gradually increases in intensity and red-shifts with respect to the LSPR band of isolated GBPs. Resulting assemblies were analyzed by Transmission Electron Microscopy (TEM) and showed a progressive elongation of the chains over time and a preferential end-to-end arrangement (Figure 1E-F and Figure S2). This structuration leads to coupling of the dipolar modes in the chain-like assembly similar to the trend observed in chains of plasmonic nanoparticles with other shapes, such as spheres and rods. ${ }^{2}$

We tuned the chain formation kinetics by adjusting the CTAB concentration in the micromolar range (Figure S3). However, without additional intervention the reaction progresses until flocculation and is impractical for further use. Fortunately, we found that the self-assembly process can be stopped at the desired stage simply by adding excess surfactant to the reaction mixture. The extinction spectra of the oligomer solutions did not evolve over 1 
month after supplementing the reaction mixture with a $10 \mathrm{mM}$ hexadecyltrimethylammonium chloride (CTAC) solution, demonstrating that the system is locked in a stable state (Figure 1C). Adding surfactant after $5 \mathrm{~h}$ of reaction yields dispersions with a predominance of the monomers plasmon band over the oligomer one. In comparison, almost no monomers remained in suspension after 20h (Figure 2B and Figure S5). This experiment shows that quenching the reaction at different time intervals allows controlling the number of GBPs per chain. Moreover, the assemblies are sturdy: they can be centrifuged and re-dispersed without affecting their optical properties (Figure S6). Overall, this strategy presents two advantages: (i) Tip-to-tip assembly simply results from a combination of salt and surfactants (with no need for stronglybinding molecules). (ii) The reaction can be frozen at any stage of the process, yielding colloidally-stable chains of the desired length.

We then explored how engineering conductive nanojunctions could control the optical properties of the nanochains by seed mediated growth. We bridged the gap between GBPs tips by injecting the assemblies as seeds in a growth solution containing a metal precursor and a mild reducing agent (ascorbic acid). In order to build nanojunction, it is important to introduce a relatively low quantity of metal (e.g. 0.2 molar equivalent Metal/Au) to avoid the formation of core-shell structures (Figure S7). Silver, palladium and platinum have been deposited in this way (Figure 2). 

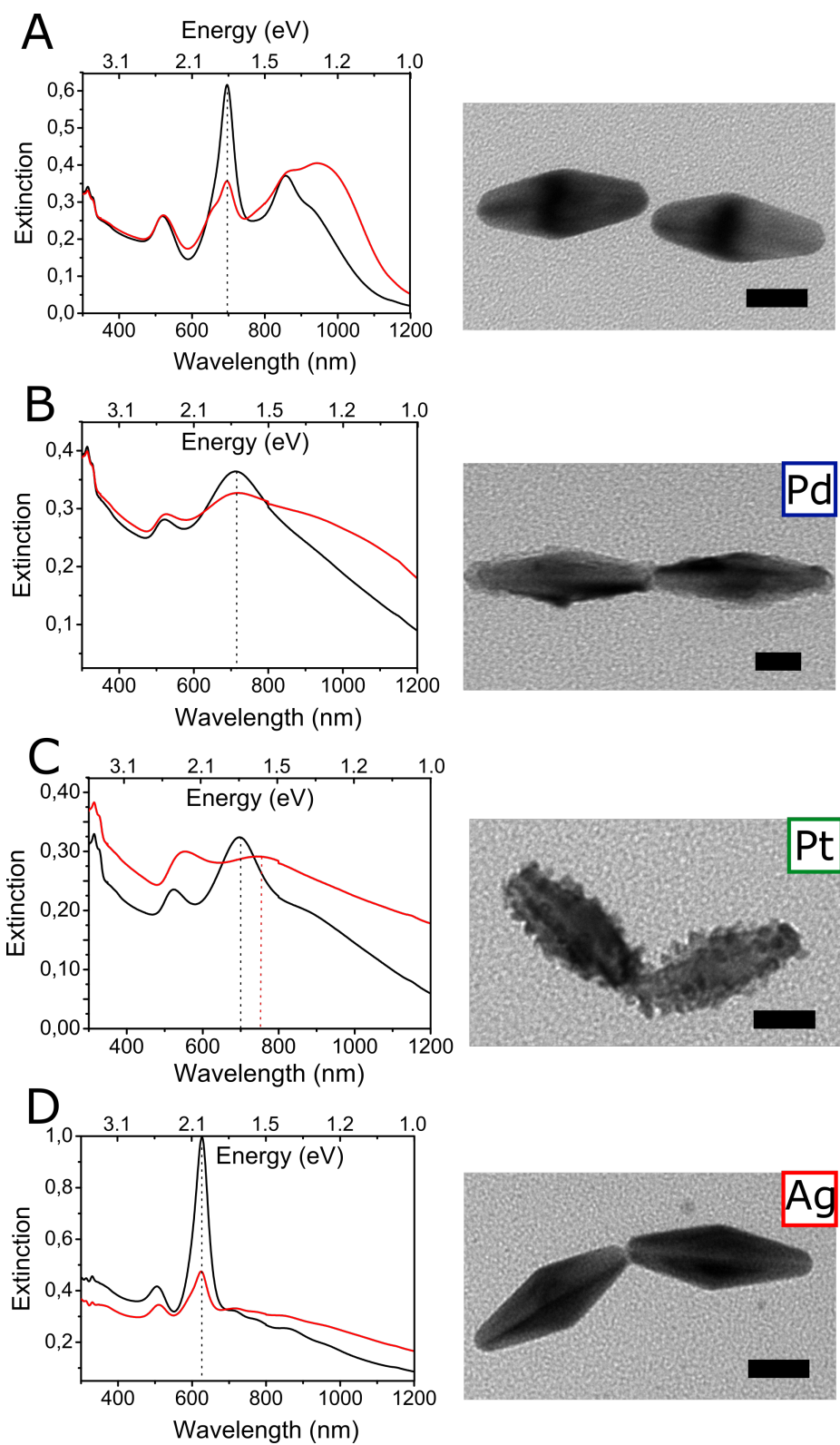

Figure 2: Tunable conductive nanojunctions in plasmonic oligomers engineered by seedmediated growth. A) UV/Vis spectra of two oligomer suspensions in which the self-assembly process was frozen after $5 \mathrm{~h}$ and $20 \mathrm{~h}$. TEM images of corresponding particles dimer. B-C) UV/Vis spectra and TEM image of dimer of the same suspensions in B) after growing nanojunctions of silver, palladium and platinum respectively. Scale bar is $20 \mathrm{~nm}$ on all images.

In the following, we denote by "oligomers" and " $\{\mathrm{Ag}, \mathrm{Pd}, \mathrm{Pt}$ or $\mathrm{Au}\}$-oligomers" the assemblies before and after the creation of the conductive nanojunctions, respectively. The formation of connected oligomers is demonstrated by the TEM images, in which the GBPs are initially 
distinct but form connected solid objects after the overgrowth reaction (Figure 2B, Figure S8). A clear evolution of the optical properties is also observed by UV/Vis spectroscopy (Figure 2B). In addition to the LSPR band corresponding to the monomers (dashed line), the suspensions exhibit two plasmon bands corresponding to the oligomers (arrows). In the case of Pd- and Pt-oligomers, the LSPR bands significantly broadens due to the interband transitions in palladium and platinum (Figure 2C-D). ${ }^{27-29}$ In contrast to palladium and platinum, silver displays a sharp plasmonic response in the visible-IR spectral range that is preserved when deposited on gold. In the case of silver, the LSPR band of the monomers at $696 \mathrm{~nm}$ shifts towards lower energies due to the deposition of thin Ag layers. ${ }^{30}$ The plasmon band of the oligomers shifted out of the visible range after connecting the NPs together. We performed electrodynamical simulation by the Boundary Element Method (BEM) using the MNPBEM17 toolbox $^{31-32}$ and the LSPR of continuous oligomers of different length was shifted systematically beyond $1200 \mathrm{~nm}$ (Figure S9). This suggests that the LSPR of the Ag-oligomers was shifted outside the experimentally accessible spectral range. Although UV/Vis measurements demonstrate a clear transition of the optical properties after connecting the particles together, it only provides information on large particle ensembles, and does not give access to the IR range relevant for long assemblies. Therefore, we used scanning transmission electron microscopy-electron energy loss spectroscopy (STEM-EELS) in order to study in more detail the collective plasmonic properties of such structures at the single particle level.

In this work, we used a NION-Hermes $200 \mathrm{~S}$ fitted with an NION-IRIS spectrometer and a Hamamatsu Kuros camera. The microscope was operated at $60 \mathrm{keV}$ with a typical $20 \mathrm{meV}$ spectral resolution and $0.5 \mathrm{~nm}$ probe size. Typical spectral-images were acquired with $5 \mathrm{~ms}$ dwell time and 15000 pixels. The dwell time was such that the zero-loss peak was not saturated. The spectra could therefore be re-aligned after acquisition. From the spectral images, either spectra or filtered map could then be extracted. In this work, spectra were obtained by summing 
around 200 pixels in a region of interest and filtered maps were typically integrated over 200 meV energy range. The sample were prepared by drop-casting the NP on a standard silicon nitride film grid. Herein, we used STEM-EELS to follow the transition of the collective modes from GBPs oligomers to Ag-oligomers and compared the results with BEM modelling (Figure 3).
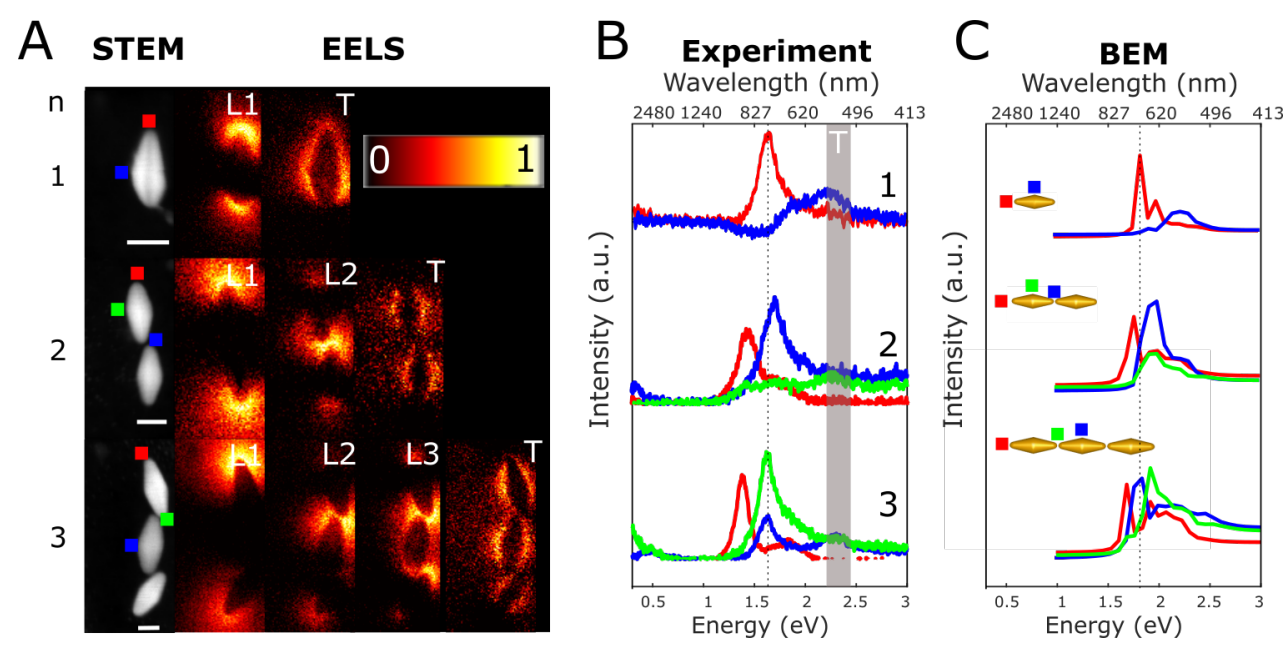

Figure 3: STEM-EELS characterization of oligomers and corresponding theoretical EELS mappings obtained by BEM. A) Spatial distribution of plasmon modes for connected oligomers as measured by EELS; Scale bar on the STEM images is $20 \mathrm{~nm}$. EELS maps are normalized to the maximum intensity in each case. B) EELS spectra taken at the location indicated by colored squares in A). C) Corresponding modeled EELS spectra taken at the location indicated by colored squares.

Single bipyramids exhibit two energy loss peaks, corresponding to the L1 and T modes centered at $1.63 \mathrm{eV}$ and $2.23 \mathrm{eV}$ respectively, in agreement with the EELS maps. As the number of particles in the chain increases, the L1 mode shifts to lower energies and new modes appear. This evolution of the plasmonic properties is well reproduced by the BEM model, even though in the simulation we considered perfectly aligned oligomers and did not account for the particles $/ \mathrm{Si}_{3} \mathrm{~N}_{4}$-membrane interaction. In the case of dimers, we observed two $\mathrm{L}_{\mathrm{N}}$ peaks, one at $1.44 \mathrm{eV}(\mathrm{L} 1)$ and $1.72 \mathrm{eV}(\mathrm{L} 2)$ that are at lower and higher energies respectively than the L1 
mode of the monomers. EELS maps confirm this attribution as the high-energy loss intensity for the L1 and L2 modes are distributed around the ends of the oligomers and at their center, respectively. This observation is consistent with the trends predicted by the plasmon hybridization theory, in which the L1 mode of the dimers correspond to the bonding mode while the L2 mode correspond to the antibonding mode. For trimers, the L1 and L2 mode are shifted to lower energies and a new L3 mode is detected. From BEM simulations, we would expect the L2 mode to have a highest loss probability around the central position (Figure S10). In the experimental EELS map, the L2 mode is localized only at one particle junction, presumably because of the lower symmetry of the nanostructure compared to the BEM model. By increasing further the number of particles in the chain, the L1 mode energy converges asymptotically to a finite value due to the so-called infinite chain limit effect $\mathrm{t}^{22,33-34}$ and the addition of new modes within a finite energy range results in a broad plasmon band. ${ }^{20}$ Those results are consistent with previous STEM-EELS studies describing the collective modes of spherical NPs chains, which observe as many $\mathrm{L}$ modes as nanoparticles in the chain. ${ }^{19-20,23}$ 

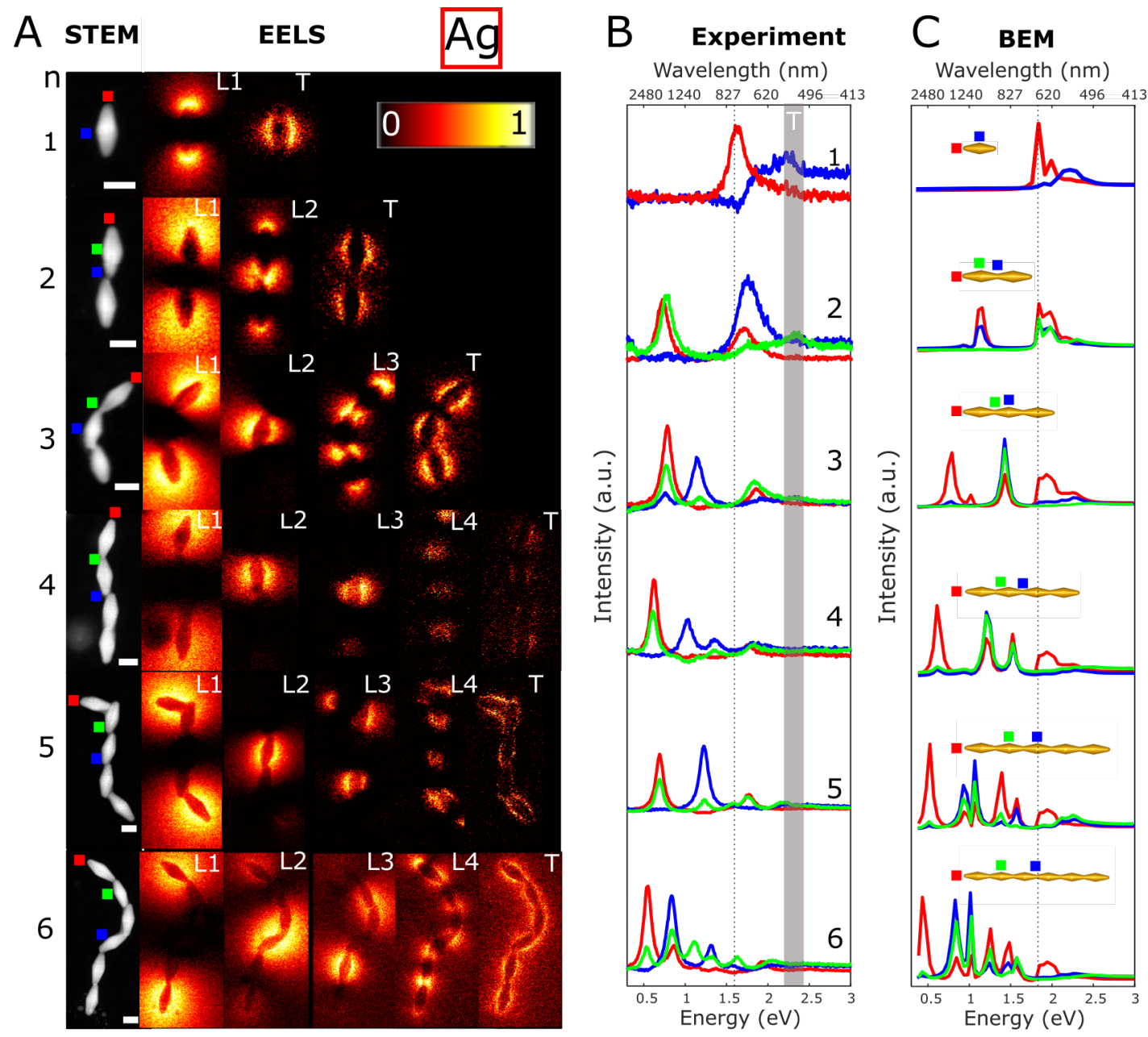

Figure 4: STEM-EELS characterization of Ag-oligomers and corresponding theoretical

EELS mappings obtained by BEM. A) Spatial distribution of plasmon modes for connected oligomers as measured by EELS; Scale bar on the STEM images is $20 \mathrm{~nm}$. EELS maps are normalized to the maximum intensity in each case. B) EELS spectra taken at the location indicated by colored squares in A). C) Corresponding BEM-modeled EELS spectra taken at the location indicated by colored squares.

Bridging the oligomers with silver has a dramatic influence on the position of the energy loss peaks (Figure 4). In the case of hexamers $(\mathrm{n}=6)$, the L1 mode energy is as low as $0.55 \mathrm{eV}$ (2250-nm equivalent free-space photon wavelength). We attribute the large spectral shifts to the transition from capacitive to conductive coupling, which allows charge transfer between neighboring NPs. ${ }^{2,8,35-36}$ The BEM calculation reproduces this evolution by modelling a 
continuous gold nanostructure (Figure 4C and Figure S11). In a control experiment, NPs in oligomers were partially fused by the electron beam induced welding to create Au-oligomers. Although Au-oligomers and Ag-oligomers were produced by two different approach, we found similar spectral and spatial distribution of the plasmon modes (Figure S12). Previous studies have shown that low-energy surface plasmon modes in Au-oligomers was resulting from the conductive junction, ${ }^{8,12,37-38}$ an interpretation that can be applied to the case of Ag-oligomers. This experiment confirms that a conducting silver bridge was deposited at the gap of Agoligomers by our approach. Note that the L1 mode does not systematically shift toward lower energies as the number of particles in the chain increases, but also depends on the symmetry of the assembly and can even shift toward higher energies in the case of oligomers that are not straight (see Figure 4 and Figure S13). The connected oligomers share similarities in terms of number, nature and energy of modes with their unconnected counterparts. For instance, the L2 modes are occurring at $1.70 \mathrm{eV}$ and $1.72 \mathrm{eV}$ in the case of dimers and connected dimers respectively. In the case of trimers, a similar loss peak energy is observed for the L3 modes at $1.85 \mathrm{eV}$ and $1.84 \mathrm{eV}$ before and after connection, respectively. This suggests that the higher $\mathrm{L}$ modes in separated and linked structures have similar physical properties, in agreement with a previous study that used templated nanostructures. ${ }^{39}$ We also performed STEM-EELS on Pdoligomers, whose L1 mode is systematically positioned between those of oligomers and Agoligomers (Figure S14). In this case, the width of the plasmon peaks is large compared to the ones observed for Ag-oligomers due to damping by interband transitions in Pd.

In summary, we connected colloidal nanoparticles by a seed-mediated growth approach that presents three original opportunities to control the properties of plasmonic dispersion. (1) The self-assembly of gold bipyramids is controlled at will in order to modulate the optical response from the visible to the NIR range. (2) By depositing silver conductive junction, plasmon are shifted towards the mid-infrared window. (3) Catalysts such as palladium and platinum can also 
be deposited at the hotspots. Although the plasmonic response is damped, such a configuration is ideal for photocatalysis because hot charge carriers would be generated in the vicinity of the catalyst. ${ }^{40}$ Similarly, our method could be applied to deposit semiconductor materials at the hotspots location to form double Schottky barriers. Overall, this benchtop method is simple, scalable and yields high-aspect-ratio nanostructures with optical properties that can be tailored across a very wide spectral range, from the visible to the mid-infrared region. We believe that this work provide a leap forward for all plasmonic application that can benefit from a precise design of the hotspots in NPs oligomers.

\section{Acknowledgements}

The CNRS is acknowledged for funding and support. Jieli Lyu acknowledges financial support by the China Scholarship Council (CSC). The present work has benefited from the electronic microscopy facility of Imagerie-Gif, (http://www.i2bc.paris-saclay.fr), member of IBiSA (http://www.ibisa.net), supported by "France-BioImaging" (ANR-10-INBS-04-01), and the Labex “Saclay Plant Science” (ANR-11-IDEX-0003-02). This work has received support from the National Agency for Research under the program of future investment TEMPOSCHROMATEM with the Reference No. ANR-10-EQPX- 50.

\section{Supporting Information}

Material and method sections, procedures for GBPs preparation, self-assembly, details on modeling, TEM images of the GBPs and corresponding UV-vis spectra, details on how to control chain formation kinetics, UV-Vis spectra of the GBPs diluted in water, UV-Vis spectra of stabilized oligomers after one month, UV-Vis spectra of the oligomers before and after centrifugation, additional TEM images of the oligomers, BEM modelling of oligomers and connected oligomers, STEM-EELS characterization of Au-connected oligomers obtained by electron beam induced welding, STEM-EELS characterization of bended nanostructures, 
STEM/EELS of Pd-connected oligomers. This material is available free of charge via the Internet at http://pubs.acs.org.

\section{References}

1. Prodan, E.; Radloff, C.; Halas, N. J.; Nordlander, P. A Hybridization Model for the Plasmon Response of Complex Nanostructures. Science 2003, 302 (5644), 419-422.

2. Halas, N. J.; Lal, S.; Chang, W.-S.; Link, S.; Nordlander, P. Plasmons in Strongly Coupled Metallic Nanostructures. Chem. Rev. 2011, 111 (6), 3913-3961.

3. Aizpurua, J.; Bryant, G. W.; Richter, L. J.; García de Abajo, F. J.; Kelley, B. K.; Mallouk, T. Optical properties of coupled metallic nanorods for field-enhanced spectroscopy. Phys. Rev. B 2005, 71 (23), 235420.

4. Sonnichsen, C.; Reinhard, B. M.; Liphardt, J.; Alivisatos, A. P. A molecular ruler based on plasmon coupling of single gold and silver nanoparticles. Nat. Biotech. 2005, 23 (6), 741-745.

5. Slaughter, L. S.; Willingham, B. A.; Chang, W.-S.; Chester, M. H.; Ogden, N.; Link, S. Toward Plasmonic Polymers. Nano Lett. 2012, 12 (8), 3967-3972.

6. Schuller, J. A.; Barnard, E. S.; Cai, W.; Jun, Y. C.; White, J. S.; Brongersma, M. L. Plasmonics for extreme light concentration and manipulation. Nat. Mater. 2010, 9 (3), 193-204.

7. Lane, L. A.; Qian, X.; Nie, S. SERS Nanoparticles in Medicine: From Label-Free Detection to Spectroscopic Tagging. Chem. Rev. 2015, 115 (19), 10489-529.

8. Zhu, W.; Esteban, R.; Borisov, A. G.; Baumberg, J. J.; Nordlander, P.; Lezec, H. J.; Aizpurua, J.; Crozier, K. B. Quantum mechanical effects in plasmonic structures with subnanometre gaps. Nat. Commun. 2016, 7, 11495.

9. Pérez-González, O.; Zabala, N.; Borisov, A. G.; Halas, N. J.; Nordlander, P.; Aizpurua, J. Optical Spectroscopy of Conductive Junctions in Plasmonic Cavities. Nano Lett. 2010, 10 (8), 3090-3095.

10. Byers, C. P.; Zhang, H.; Swearer, D. F.; Yorulmaz, M.; Hoener, B. S.; Huang, D.; Hoggard, A.; Chang, W.-S.; Mulvaney, P.; Ringe, E.; Halas, N. J.; Nordlander, P.; Link, S.; Landes, C. F. From tunable core-shell nanoparticles to plasmonic drawbridges: Active control of nanoparticle optical properties. Sci. Adv. 2015, 1 (11), e1500988.

11. Hoener, B. S.; Kirchner, S. R.; Heiderscheit, T. S.; Collins, S. S. E.; Chang, W.-S.; Link, S.; Landes, C. F. Plasmonic Sensing and Control of Single-Nanoparticle Electrochemistry. Chem. 2018, 4 (7), 1560-1585.

12. Teulle, A.; Bosman, M.; Girard, C.; Gurunatha, K. L.; Li, M.; Mann, S.; Dujardin, E. Multimodal plasmonics in fused colloidal networks. Nat. Mater. 2015, 14 (1), 87-94.

13. González-Rubio, G.; González-Izquierdo, J.; Bañares, L.; Tardajos, G.; Rivera, A.; Altantzis, T.; Bals, S.; Peña-Rodríguez, O.; Guerrero-Martínez, A.; Liz-Marzán, L. M. Femtosecond Laser-Controlled Tip-to-Tip Assembly and Welding of Gold Nanorods. Nano Lett. 2015, 15 (12), 8282-8288. 
14. Herrmann, L. O.; Valev, V. K.; Tserkezis, C.; Barnard, J. S.; Kasera, S.; Scherman, O. A.; Aizpurua, J.; Baumberg, J. J. Threading plasmonic nanoparticle strings with light. Nat. Commun. 2014, 5, 4568.

15. Fang, L.; Wang, Y.; Liu, M.; Gong, M.; Xu, A.; Deng, Z. Dry Sintering Meets Wet SilverIon "Soldering": Charge-Transfer Plasmon Engineering of Solution-Assembled Gold Nanodimers From Visible to Near-Infrared I and II Regions. Angew. Chem. Int. Ed. 2016, 55 (46), 14296-14300.

16. Lee, J.-H.; You, M.-H.; Kim, G.-H.; Nam, J.-M. Plasmonic Nanosnowmen with a Conductive Junction as Highly Tunable Nanoantenna Structures and Sensitive, Quantitative and Multiplexable Surface-Enhanced Raman Scattering Probes. Nano Lett. 2014, 14 (11), 6217 6225 .

17. Kociak, M.; Stephan, O. Mapping plasmons at the nanometer scale in an electron microscope. Chem. Soc. Rev. 2014, 43 (11), 3865-3883.

18. Nelayah, J.; Kociak, M.; Stephan, O.; Garcia de Abajo, F. J.; Tence, M.; Henrard, L.; Taverna, D.; Pastoriza-Santos, I.; Liz-Marzan, L. M.; Colliex, C. Mapping surface plasmons on a single metallic nanoparticle. Nat. Phys. 2007, 3 (5), 348-353.

19. Barrow, S. J.; Rossouw, D.; Funston, A. M.; Botton, G. A.; Mulvaney, P. Mapping Bright and Dark Modes in Gold Nanoparticle Chains using Electron Energy Loss Spectroscopy. Nano Lett. 2014, 14 (7), 3799-3808.

20. Mayer, M.; Potapov, P. L.; Pohl, D.; Steiner, A. M.; Schultz, J.; Rellinghaus, B.; Lubk, A.; Konig, T. A. F.; Fery, A. Direct Observation of Plasmon Band Formation and Delocalization in Quasi-Infinite Nanoparticle Chains. Nano Lett. 2019, 19 (6), 3854-3862.

21. Koh, A. L.; Bao, K.; Khan, I.; Smith, W. E.; Kothleitner, G.; Nordlander, P.; Maier, S. A.; McComb, D. W. Electron Energy-Loss Spectroscopy (EELS) of Surface Plasmons in Single Silver Nanoparticles and Dimers: Influence of Beam Damage and Mapping of Dark Modes. ACS Nano 2009, 3 (10), 3015-3022.

22. Willingham, B.; Link, S. Energy transport in metal nanoparticle chains via sub-radiant plasmon modes. Opt. Express 2011, 19 (7), 6450-6461.

23. Barrow, S. J.; Collins, S. M.; Rossouw, D.; Funston, A. M.; Botton, G. A.; Midgley, P. A.; Mulvaney, P. Electron Energy Loss Spectroscopy Investigation into Symmetry in Gold Trimer and Tetramer Plasmonic Nanoparticle Structures. ACS Nano 2016, 10 (9), 8552-8563.

24. Liu, K.; Nie, Z.; Zhao, N.; Li, W.; Rubinstein, M.; Kumacheva, E. Step-Growth Polymerization of Inorganic Nanoparticles. Science 2010, 329 (5988), 197-200.

25. Guerrero-Martinez, A.; Grzelczak, M.; Liz-Marzan, L. M. Molecular thinking for nanoplasmonic design. ACS Nano 2012, 6 (5), 3655-62.

26. Abtahi, S. M. H.; Burrows, N. D.; Idesis, F. A.; Murphy, C. J.; Saleh, N. B.; Vikesland, P. J. Sulfate-Mediated End-to-End Assembly of Gold Nanorods. Langmuir 2017, 33 (6), 14861495.

27. Khurgin, J. B.; Boltasseva, A. Reflecting upon the losses in plasmonics and metamaterials. MRS Bull. 2012, 37 (8), 768-779.

28. Langhammer, C.; Yuan, Z.; Zorić, I.; Kasemo, B. Plasmonic Properties of Supported Pt and Pd Nanostructures. Nano Lett. 2006, 6 (4), 833-838. 
29. Garcia, M. A. Surface plasmons in metallic nanoparticles: fundamentals and applications. J. Phys. D Appl. Phys. 2011, 44 (28), 283001.

30. Dacosta Fernandes, B.; Spuch-Calvar, M.; Baida, H.; Tréguer-Delapierre, M.; Oberlé, J.; Langot, P.; Burgin, J. Acoustic Vibrations of Au Nano-Bipyramids and their Modification under Ag Deposition: a Perspective for the Development of Nanobalances. ACS Nano 2013, 7 (9), 7630-7639.

31. Hohenester, U. Simulating electron energy loss spectroscopy with the MNPBEM toolbox. Comput. Phys. Commun. 2014, 185 (3), 1177-1187.

32. Hohenester, U.; Trügler, A. MNPBEM - A Matlab toolbox for the simulation of plasmonic nanoparticles. Comput. Phys. Commun. 2012, 183 (2), 370-381.

33. Barrow, S. J.; Funston, A. M.; Gómez, D. E.; Davis, T. J.; Mulvaney, P. Surface Plasmon Resonances in Strongly Coupled Gold Nanosphere Chains from Monomer to Hexamer. Nano Lett. 2011, 11 (10), 4180-4187.

34. Citrin, D. S. Plasmon Polaritons in Finite-Length Metal-Nanoparticle Chains: The Role of Chain Length Unravelled. Nano Lett. 2005, 5 (5), 985-989.

35. Kadkhodazadeh, S.; Wagner, J. B.; Kneipp, H.; Kneipp, K. Coexistence of classical and quantum plasmonics in large plasmonic structures with subnanometer gaps. Applied Physics Lett. 2013, 103 (8), 083103.

36. Alber, I.; Sigle, W.; Demming-Janssen, F.; Neumann, R.; Trautmann, C.; van Aken, P. A.; Toimil-Molares, M. E. Multipole Surface Plasmon Resonances in Conductively Coupled Metal Nanowire Dimers. ACS Nano 2012, 6 (11), 9711-9717.

37. Su, M.-N.; Sun, Q.; Ueno, K.; Chang, W.-S.; Misawa, H.; Link, S. Optical Characterization of Gold Nanoblock Dimers: From Capacitive Coupling to Charge Transfer Plasmons and Rod Modes. J. Phys. Chem. C 2018, 122 (31), 18005-18011.

38. Wen, F.; Zhang, Y.; Gottheim, S.; King, N. S.; Nordlander, P.; Halas, N. J. Charge Transfer Plasmons: Optical Frequency Conductances and Tunable Infrared Resonances. ACS Nano 2015, 9 (6), 6428-35.

39. Osberg, K. D.; Harris, N.; Ozel, T.; Ku, J. C.; Schatz, G. C.; Mirkin, C. A. Systematic Study of Antibonding Modes in Gold Nanorod Dimers and Trimers. Nano Lett. 2014, 14 (12), 69496954.

40. Besteiro, L. V.; Kong, X.-T.; Wang, Z.; Hartland, G.; Govorov, A. O. Understanding HotElectron Generation and Plasmon Relaxation in Metal Nanocrystals: Quantum and Classical Mechanisms. ACS Photonics 2017, 4 (11), 2759-2781. 